\title{
Crafting Audience Engagement in Social Media Conversations: Evidence from the U.S. 2020 Presidential Elections
}

\author{
Linus Hagemann \\ University of Potsdam \\ linus.hagemann@uni-potsdam.de
}

\author{
Olga Abramova \\ University of Potsdam \\ olga.abramova@uni-potsdam.de
}

\begin{abstract}
Observing inconsistent results in prior studies, this paper applies the elaboration likelihood model to investigate the impact of affective and cognitive cues embedded in social media messages on audience engagement during a political event. Leveraging a rich dataset in the context of the 2020 U.S. presidential elections containing more than 3 million tweets, we found the prominence of both cue types. For the overall sample, positivity and sentiment are negatively related to engagement. In contrast, the post-hoc subsample analysis of tweets from famous users shows that emotionally charged content is more engaging. The role of sentiment decreases when the number of followers grows and ultimately becomes insignificant for Twitter participants with a vast number of followers. Prosocial orientation ("we-talk") is consistently associated with more likes, comments, and retweets in the overall sample and sub-samples.
\end{abstract}

\section{Introduction}

"Don't ever diminish the power of words. Words move hearts, and hearts move limbs." Hamza Yusuf

Today, social media is firmly sewed into the public discourse and permanently accompanies civic and political life (for meta-review, see [1]), e.g., events like festivals, demonstrations [2], elections [3], as well as crises [4]. In the past decade, the popularity of Twitter, Facebook, Instagram, and, more recently, TikTok skyrocketed and turned these platforms into digital "barometers" of the community's mood and intentions, simultaneously representing the shortest and most straightforward way to reach and convince an audience [5].

Consequently, knowledgeable information senders pay a lot of attention to crafting social media content. Indeed, extensive evidence suggests that linguistic features matter for acceptance and sharing of news articles [6][7], the support of crowdfunding projects [8], or patients in online health communities (OHC) [9]. Among others, ICT-mediated conversations in the context of political events received significant attention [10][3]. Especially Twitter has established itself as a forum for political deliberation, with posts validly mirroring offline political sentiment [5]. Noteworthy is the swiftness of information dissemination: based on Twitter data on the 2016 E.U. referendum ("Brexit") and the 2016 U.S. presidential elections, [11] finds that information diffusion on this SNS is largely complete within 1-2 hours. The speed and the engagement of a big audience are essential to reap the benefits of Twitter as a medium of collective movement, e.g., when organizing protests or reacting to crisis events [12].

In spite of multiple scholarly attempts to connect message sentiment and audience engagement in terms of feedback and willingness to share [3][4], a closer examination of their results shows mixed evidence (see Section 2), even within the political domain. Concept-wise, most past studies take a one-sided perspective and focus on the affective dimension of the message, i.e., sentiment valence and intensity, often ignoring the cognitive dimension. Meanwhile, it has been shown that both affective and cognitive aspects are complementary in involving the audience [9] [13]. Against this background, we ask:

RQ: How are social media message characteristics related to audience engagement?

To answer this question, we lean on the elaboration likelihood model [13] as the overarching theoretical framework and conceptualize public social media posts as a way to communicate persuasive messages [14]. Our research model combines cues on the central and peripheral route, including sentiment valence, intensity, and social orientation, to explain social engagement in computer-mediated conversations, operationalized via likes, shares, and comments as typical feedback opportunities online.

To empirically examine our research propositions, we leverage a novel dataset of tweets made in the context of the 2020 U.S. presidential 
elections. In this high-stake setting, social media is a scene to inform, persuade, ultimately gain support and thus more votes [15]. Political candidates present themselves and their vision, while citizens can react to it or express their own opinions on issues, possibly tipping the scales. Thus, the selected research site provides a solid ground to examine the research question stated above.

We report the following set of results. First, we find that on the overall sample, both central route (cognitive) and peripheral route (affective) cues matter for the audience engagement and information dissemination. Second, we revealed that the importance of sentiment (i.e., emotional charge) decreases with the increasing popularity of the author (negative moderation), becoming insignificant for people with more than 1 million followers. We also find the significance of prosocial orientation expressed in tweets ("we-talk") created positive feedback in terms of likes, comments, and retweets. "I-talk" is beneficial only for famous people with many followers, while for an average user, it might be harmful and lead to fewer likes, comments, and shares. Moreover, cognitive cues exhibited their prominence, with language indicating discrepancy being positively related to the overall engagement. For tweets written by a famous person, expressed certainty in the used language is desirable.

Our work makes several contributions to the academic literature and practice. Building on prior studies that have examined the effects of the sentiment of social media content [10][3], we extend the literature by empirical evidence derived from a recent large-scale political event such as the U.S. presidential elections. Whereas earlier works conceptually focus on the impact of the peripheral route cues [6][10], our model exhibits the effects of both central and peripheral route cues.

By demonstrating that tweet characteristics have different impacts in terms of audience feedback for famous people with millions of followers as compared to the overall Twitter audience, our study contributes to the current literature on the role of popularity and influence in the social media space. From the practice perspective, our results hint at the necessity to craft the messages by famous people differently from messages by average users when one wants to maximize engagement.

\section{Theoretical background}

This paper looks at social media as a stimulibased environment, widely used to form and influence opinions about products, services, and events. Stimuli are represented through text, video, images, audio, animations, or a layout [13]. Especially during a political event, social media conversations aim to intrigue, promote an opinion, inform, convince, or call for action and therefore can be conceptualized as persuasive messages [14]. In the last decade, instant posts on Twitter or Facebook have become the primary tool to spread ideas, find supporters, and observe the electorate mood [10]. Thus, every message represents a persuasion opportunity for an information sender, which may influence recipients by drawing their attention, assigning cognitive resources, and evoking affective responses and behaviors [13].

The elaboration likelihood model (ELM) proposes that when processing persuasive messages, a recipient engages in elaboration approaches [16]. If a recipient possesses motivation and skills to handle analytical information, they engage in central route processing [13]. It implies decisions grounded in careful consideration of logic and reason, researching relevant facts, and imposes high demands on cognitive resources [14]. In the lack of either motivation or skills to regard analytical information, the processing happens via the peripheral route [13]. This method is less effortful, and judgments come down to heuristics, rules of thumb, habits, impulses, emotions, and desires [14]. On social media, because the audience is highly heterogeneous and unknown, information senders are interested in what stimuli work best for each route to ensure comprehensive audience coverage.

Perceiving prior investigations through the ELM lens, we observe extensive evidence on the effects of message characteristics processed in the peripheral route. Studies are aligned that emotionally intensive content is shared more often in news settings [6][7] and political discourse [10]. At the same time, a closer look at the results reveals inconsistencies, even accounting for the context. E.g., when it comes to a message valence (i.e., whether it is emotionally positive, negative, or neutral) and its association with sharing behavior, the evidence is mixed. Exhibiting that positively charged news articles in prominent U.S. and German electronic outlets are more frequently emailed, shared with SNS friends on Facebook, Twitter, and Google+, and receive feedback with the "Like" button, [6] and [7] advocate for the so-called positivity bias. Further, an upbeat emotional language style increased information sharing during a crisis event [17]. Contrary to them, in online health communities (OHC), more negative content receives greater support [9], confirming a negativity bias. Political tweets having a negative sentiment were insignificantly related to retweeting frequency and speed in [10]. A recent paper by R. Mallipeddi [3] submits that both positive and negative toned tweet content by candidates in the Indian election is 
associated with higher engagement operationalized by retweet numbers. Theory-wise, [9] represents an exception regarding both central and peripheral route cues. Taken together, the lack of consensus among prior studies, as well as the dominance of a one-sided perspective to social media message processing (by focusing on the peripheral route), is a research gap, which motivated our current investigation.

\section{Research model and hypotheses}

Under the ELM, engagement can be achieved by logical argumentation or emotional arousal of the audience. We start building our research model (Figure 1) with the path less effortful for human cognition, i.e., message peculiarities that may drive persuasion through the peripheral route.

\subsection{Peripheral route of persuasion in political SNS conversations}

Humans have an evolutionally rooted proclivity to pay attention to negative information rather than positive information. The amygdala, the almondshaped brain structure responsible for the alertness to danger, employs two-thirds of its neurons to search for "bad" stimuli, and upon recognition, quickly store them in memory, activating the ancient fight-or-flight limbic system [18]. Positive experiences "usually need to be held in awareness for a dozen or more seconds to transfer from short-term memory buffers to long-term storage." [18]. Not only do adverse events imprint quicker, but they also linger longer - a tendency known as negativity bias, which has been consistently observed across domains and nations [19]. In mediated communication, a more vivid reaction to negative content was found for online reviews [20], video news [19], and political Facebook posts [21]. We assume the negativity bias to hold in political Twitter communication, with negative content producing more feedback. Thus, we hypothesize:

H1: The lower the positivity (a.k.a. emotional valence) expressed in a SM post during a political event, the higher engagement it produces.

Perceiving new information as a persuasion signal, a stronger signal should produce a more intensive response. For online conversations, past studies suggest that highly emotionally charged messages generate more feedback than neutral ones [10][9]. In line with this, we hypothesize:

H2: The higher the sentiment (a.k.a. emotional intensity) expressed in a SM post during a political event, the higher engagement it produces.
In addition to emotional valence and its intensity, the feeling of affiliation matters in social media conversations as well. Evolutionally, belongingness to a group as a bigger, stronger entity compared to a single individual, creates a sense of safety and is therefore valuable. Nowadays, the feeling of togetherness is associated with healthy relationship behaviors, e.g., being kind and empathetic in stressful times. Interdependence theory states that interdependent romantic partners are more inclusive in how they both think, feel, behave, and rely on each other for the support over time [22]. Linguistically, the interdependence is expressed in the frequent use of $1^{\text {st }}$ person plural pronouns, a.k.a. we-talk, implying that a speaker includes a partner in the events, planning, and experiences. A meta-analysis of 30 studies confirmed couples who often say "we" and "us" have better relationship outcomes, as well as better mental and physical health for people in romantic relationships [23]. In the political context, the royal "we" is commonly employed by monarchs, bishops, and university rectors since they are often speaking as leaders of a nation or institution. A multi-method investigation across 5 studies found that participants with higher status consistently used fewer $1^{\text {st }}$-person singular (I) and more 1st-person plural (we) and $2^{\text {nd }}$ person singular (you) pronouns in both writing and speech [24]. The researchers attributed this pattern to the demonstration of a greater focus on others, using "we" in a prosocial way [25]. If this reasoning holds, the audience should be engaged more with posts that show a carrying attitude. Thus, we hypothesize:

H3: The higher the prosocial orientation expressed in a SM post during a political event, the higher engagement it produces.

\subsection{Central route of persuasion in political SNS conversations}

We surmise that in political conversations, not only an emotional but also an analytical part is important [26], for which evaluation happens via the central route. Staying informed on current events is one motivation of many social media users [26]. Especially younger generations consider SNSs as a source of political knowledge [27]. Past studies in the political context show that social media platforms foster the exchange of information that is vital for coordination and collective action [28][29]. Therefore, we hypothesize:

H4: The higher the insight expressed in a SM post during a political event, the higher engagement it produces.

People continually rely on causation when clarifying what has already happened, to foresee what 
will come, and to influence what happens in the future [30]. The persuasive power of causal arguments is known in social psychology [31] and consumer research [32]. In politics, causal claims help to comprehend the range of alternatives available to national and international actors, to develop policy prescriptions, and to critically assess policy decisions against other feasible solutions [15]. During the U.S. presidential elections, the stakes are high for the candidates, their parties, U.S. citizens, and beyond. Not only the concrete proposals are of interest, but the reasoning behind them, which allow voters to anticipate the consequences of their decision in the long term [32]. Thus, we hypothesize:

H5: The higher the causation expressed in a SM post during a political event, the higher engagement it produces.

Leadership is often related to confidence. Decreased confidence is perceived as a warning signal in many sectors, including religion and business, from education to nonprofits [33]. We expect strong, clear opinions to get a lot of positive reactions from the people that agree with them while also being a point of vivid discussion for people disagreeing. Expressions made with a high degree of certainty have the potentials to be polarizing [34]. Therefore, our hypothesis is:

H6: The higher the certainty expressed in a SM post during a political event, the higher engagement it produces.

Spotting discrepancies between how a situation is now and how it possibly should be in the future is critical for communicating a strategic plan, which, in turn, is attractive and empowering [35]. Polemic on hopes, vision, needs, fears, and expectations one has for the outcome of the election and thus, the next government typically produce a resonance. In line with our reasoning, we expect those to be an essential topic of political discussions on election day when SM users are likely to share their personal opinions and beliefs in order to convince still undecided voters. Hence, we hypothesize:

H7: The higher the discrepancy expressed in a SM post during a political event, the higher engagement it produces.

In the heterogeneous SM audience, one can distinguish popular users, i.e., online celebrities with many followers, commonly coined influencers [36]. Influencers are recognized as experts in a field (e.g., politics, culture, entertainment industry) and already enjoy public attention [36]. Due to reputation, posts by highly followed people are likely to produce more resonance (e.g., retweets [10]) than those of an ordinary information sender regardless of their content. Following [3], who found a negative moderating effect of popularity on the relationship between positive sentiment and engagement for the Indian election in 2014, we suspect that online fame may intrude upon the above links stemming from the ELM and hypothesize:

H8: The relationship between a SM post's characteristics and the engagement produced is weaker for SM users with a large number of followers.

Figure 1 summarizes the proposed hypotheses.

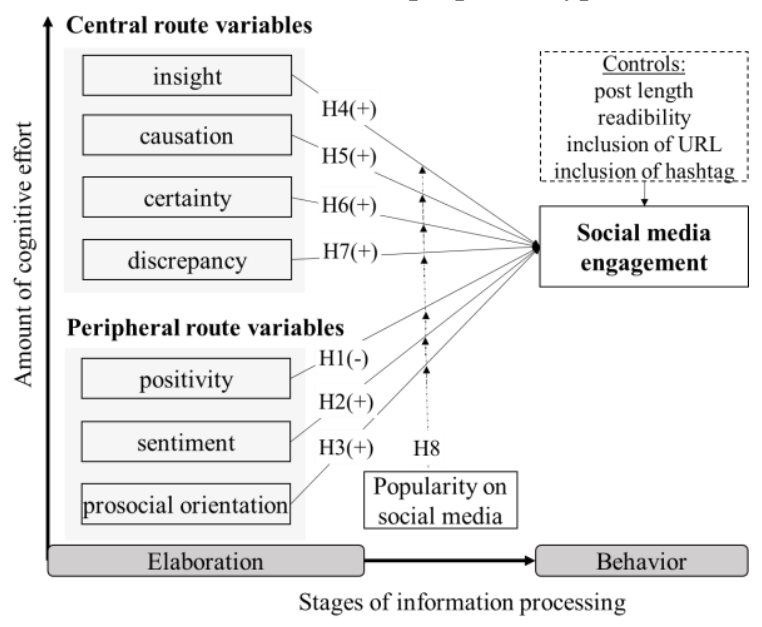

Figure 1. Research model

\section{Data and methodology}

To empirically test our propositions, we created a comprehensive dataset of over 3 million posts on Twitter written in English on November 03, 2020, the day of the U.S. Presidential Elections. We considered tweets made on November 03 in any U.S. mainland time zone. Using the web-scraping tool twint, the following hashtags were searched: \#biden, \#trump, \#republicans, \#democrats, \#2020election, and \#USelection. We expect that on election day, social media interactions are especially intense as each side tries to utilize the last moments available to convince voters that are still undecided and raise the spirits of initial supporters.

We performed several cleaning steps on our corpus. Precisely, special characters (e.g., \#, /, @) were removed from the tweet contents, as well as URLs and e-mail addresses. Following [10], we controlled for the existence of hashtags and URLs by introducing variables that indicate the presence of a URL in the original tweet and the number of hashtags, respectively, since the abundance of those might be an indicator of questionable content like spam. Language detection was performed on the tweets using the python package langdetect, and all tweets that were not classified as English were not considered for the final data set. Langdetect utilizes a naïve Bayesian 
filter to detect language based on character n-grams [37]. This was done as a precaution to remove tweets that might have been wrongly returned when scraping to reduce the possibility of errors during the later language and sentiment analysis, which are dictionarybased. Finally, duplicate tweets were eliminated. After cleaning, the final dataset comprises 3,172,976 unique tweets made by 1,405,284 unique Twitter users, of which 512,796 posted multiple times.

\subsection{Variables}

In this study, social media engagement is operationalized through the number of replies to a post, i.e., the number of likes, comments, and shares (i.e., retweets). Though all these actions indicate public reactions, they might differ in meaning. Thus, clicking on the "like" button is a form of personal feedback, which expresses agreement and full support, and requires the least effort. Commenting requires more effort and can express any feedback, including approval (agreement) or rejection (disagreement). Resharing is seen to be the most effortful action on the side of the information recipient.

The strength of positive/negative emotions expressed in the Twitter posts was assessed with the sentiment analysis tool SentiStrength [38], exhibited to outperform other machine learning approaches [39] and deemed to be state of the art for the analysis of tweets [40]. To each tweet, SentiStrength assigns a positive sentiment score, which ranges from 1 (no positive attitude) to 5 (very strong positive attitude), and a negative sentiment score, on a scale from -1 (no negative attitude) to -5 (very strong negative attitude). Following Stieglitz and Dang-Xuan [10], we applied the formula (positive score - negative score) -2 to derive the variable sentiment (Senti), which reflects the extent of overall emotional charge and varies on a scale from 0 (low emotional intensity) to 8 (high emotional intensity). Likewise, the degree of positivity (Pos) is defined as (positive score + negative score + 4 ) and ranges from 0 (very negative content) to 8 (very positive content) [10].

Prosocial orientation and cognitive cues were assessed using the linguistic inquiry and word count (LIWC) software tool [41]. LIWC calculates the percentage of particular words in the text, so its measures are always relative to the length of the message. Precisely, prosocial orientation was measured as the proportion of $1^{\text {st }}$ person plural pronouns (we, our) and contrasted with $1^{\text {st }}$ person single pronouns (I, my) and $3^{\text {rd }}$ person plural pronouns (they, their). Cognitive processes were operationalized as the percentage of words expressing insight (Ins) (e.g., think, know), causation (Cause) (e.g., because, effect), certainty (Cert) (e.g., always, never), and discrepancy (Disc) (e.g., should, would) [41].

Social media popularity expressed in the number of followers an account has is addressed via the fame level proxy. Based on lists of influential Twitter accounts in the U.S. in the domains of celebrities $(n=1,000)$, media and news outlets $(n=1,000)$, and politicians $(n=639)$ from Socialbakers [42], we collected influential accounts and their follower numbers. With this, we assigned each tweet in our sample a fame level (Fame) using the following criteria. Level: 0 - account is not present on the list; 1 - account is present on the list and has <100,000 followers; 2 - account is present on the list and has more than 100,000 and less than 1,000,000 followers; 3 - account is present on the list and has more than $1,000,000$. For each account, the highest fitting category was assigned.

We control for the post length, i.e., the number of words in a post. Since past research reports the significance of URL and hashtag inclusion (e.g., [43], [10]), the existence of at least one URL in a post (URL) and the number of hashtags in a post were also regarded (hashtags). User activity, expressed as the number of tweets a given user posted in our sample, is included to control for excessive posting, which, e.g., could indicate social bots.

For the target variables that count likes, retweets, and the number of comments a post achieved, outliers were removed from the sample. The decision was made based on boxplots of the respective variables. Therefore, e.g., a post made by the former U.S. president Barack Obama was excluded, as his exceptionally high numbers of followers $(\mathrm{N}=129,774,783$ on June 13,2021$)$ led to an extreme number of interactions (210,942 likes, 8,393 comments, and 24,467 retweets).

The unit of analysis was the single Twitter post (a tweet). On average, a tweet in our sample has received 20 likes, three retweets, and one reply. Standard deviations for all these variables in the complete sample are high, inter alia, because tweets made from accounts with a fame level greater than zero got 1,571 likes, 204 retweets, and 77 replies on average. The highest user activity we found was 1,482 tweets made on election day in the overall sample and 333 tweets posted from an account classified as famous. We provide our final data set as well as all code used for the collection, preprocessing, and analysis of the data in a GitHub repository ${ }^{1}$.

\footnotetext{
${ }^{1}$ https://github.com/linusha/twitter-sentiment-2020-election
} 
Table 1. Regression estimates for the overall sample

\begin{tabular}{|c|c|c|c|c|c|c|}
\hline Model & (1) & (2) & (3) & (4) & (5) & (6) \\
\hline Method & \multicolumn{3}{|c|}{ Negative binomial regression } & \multicolumn{3}{|c|}{ Logistic regression } \\
\hline $\mathrm{DV} \rightarrow$ & LIKE & $\mathrm{COM}$ & RET & LIKE & $\mathrm{COM}$ & RET \\
\hline Positivity & $-0.019 * * *$ & $-0.031 * * *$ & $-0.029 * * *$ & $0.009 * *(0.001)$ & $-0.019 * *(0.001)$ & $-0.014 * *(0.001)$ \\
\hline Sentiment & $-0.112 * * *$ & $-0.101 * * *$ & $-0.130 * * *$ & $-0.024 * *(0.001)$ & $-0.119 * *(0.001)$ & $-0.019 * *(0.001)$ \\
\hline I-talk & $-0.018 * * *$ & $0.004 * * *$ & $-0.031 * * *$ & $0.030 * *(0.0003)$ & $0.018 * *(0.0003)$ & $-0.002 * *(0.0004)$ \\
\hline We-talk & $0.005 * * *$ & $-0.004 * * *$ & $0.004 * * *$ & $0.009 * *(0.0004)$ & $-0.020 * *(0.001)$ & $0.016 * *(0.001)$ \\
\hline They-talk & $-0.016 * * *$ & $-0.025 * * *$ & $-0.003 *$ & $0.003 * *(0.001)$ & $-0.004 * *(0.001)$ & $-0.002 *(0.001)$ \\
\hline Insight & $-0.021 * * *$ & $0.003 * * *$ & $-0.038 * * *$ & $-0.002 * *(0.0004)$ & $0.013 * *(0.0004)$ & $-0.014 * *(0.001)$ \\
\hline Cause & $0.003 * * *$ & $0.003 * * *$ & $0.012 * * *$ & $0.003 * *(0.0004)$ & $0.012 * *(0.0004)$ & $0.002 * *(0.001)$ \\
\hline Discrepancy & $0.001 * * *$ & $0.016 * * *$ & $0.023 * * *$ & $0.015 * *(0.0003)$ & $0.005 * *(0.0004)$ & $0.015 * *(0.0005)$ \\
\hline Certainty & $-0.008 * * *$ & $-0.018 * * *$ & $-0.018 * * *$ & $-0.001 *(0.0004)$ & $-0.011 * *(0.0004)$ & $-0.001 *(0.001)$ \\
\hline $\mathrm{N}$ hashtags & $-0.105 * * *$ & $-0.093 * * *$ & $-0.067 * * *$ & $0.007 * *(0.001)$ & $-0.145 * *(0.002)$ & $0.084 * *(0.001)$ \\
\hline URL & $-0.301 * * *$ & $0.066^{* * *}$ & $0.053 * * *$ & $-0.507 * *(0.005)$ & $-0.595 * *(0.006)$ & $0.593 * *(0.006)$ \\
\hline WC & $0.021 * * *$ & $0.021 * * *$ & $0.029 * * *$ & $0.009 * *(0.0001)$ & $0.025 * *(0.0001)$ & $0.012 * *(0.0001)$ \\
\hline User activity & $0.001 * * *$ & $0.009 * * *$ & $0.010 * * *$ & $-0.001 * *(0.00003)$ & $-0.001 * *(0.00003)$ & $-0.00000(0.00003)$ \\
\hline Fame 1 & 0.045 & 0.813 & 0.158 & $1.396(1.955)$ & $2,569 * *(0.578)$ & $0.482(0.846)$ \\
\hline Fame 2 & $2.348 * * *$ & $2.060 * * *$ & $1.686 * * *$ & $3.968 * *(0.393)$ & $2.601 * *(0.172)$ & $2.704 * *(0.190)$ \\
\hline Fame 3 & $4.009 * * *$ & $4.141 * * *$ & $3.646 * * *$ & $7.700 * *(1.5)$ & $6.011 * *(0.480)$ & $6.520 * *(0.650)$ \\
\hline Fame $1 *$ Pos & 0.245 & 0.201 & 0.316 & $0.225(0.479)$ & $-0.174(0,128)$ & $0.403 *(0.200)$ \\
\hline Fame $2 *$ Pos & -0.091 & -0.048 & 0.031 & $0.071(0.094)$ & $0.011(0.041)$ & $0.090 *(0.046)$ \\
\hline Fame $3 *$ Pos & 0.069 & 0.039 & 0.086 & $-0.128(0.354)$ & $-0.163(0.113)$ & $-0.056(0.151)$ \\
\hline Fame $1 *$ Senti & 0.233 & 0.114 & 0.224 & $1.443 * *(0.499)$ & $0.166(0.130)$ & $1.176 * *(0.220)$ \\
\hline Fame $2 *$ Senti & $0.292 * * *$ & $0.247 * * *$ & $0.347 * * *$ & $-0.059(0.087)$ & $0.171 * *(0.039)$ & $0.078^{\dagger}(0.043)$ \\
\hline Fame $3 *$ Senti & $0.128^{*}$ & 0.062 & 0.172 & $-0.238(0.301)$ & $-0.033(0.097)$ & $-0.020(0.133)$ \\
\hline Fame $1 * \mathrm{I}$ & 0.045 & -0.005 & -0.055 & $-0.056(0.098)$ & $0.022(0.058)$ & $-0.046(0.060)$ \\
\hline Fame $2 * I$ & $0.136 * * *$ & $0.118^{* * *}$ & 0.126 & $2.686(5.322)$ & $0.143 * *(0.053)$ & $0.172 * *(0.055)$ \\
\hline Fame $3 *$ I & $0.194 * * *$ & $0.149 * * *$ & $0.186^{* *}$ & $-0.184^{\dagger}(0.106)$ & $-0.051(0.059)$ & $-0.133 * *(0.043)$ \\
\hline Fame $1 * \mathrm{We}$ & 0.019 & -0.076 & 0.023 & $-0.039(0.106)$ & $0.010(0.51)$ & $0.004(0.059)$ \\
\hline Fame $2 * \mathrm{We}$ & 0.015 & $0.085 * * *$ & 0.004 & $-0.148 * *(0.024)$ & $-0.037 * *(0.015)$ & $-0.039 *(0.017)$ \\
\hline Fame $3 * \mathrm{We}$ & $0.161 * * *$ & $0.099 * * *$ & $0.140 * * *$ & $-0.064(0.123)$ & $0.039(0.044)$ & $0.008(0.067)$ \\
\hline Fame $1 *$ They & 0.145 & 0.235 & 0.260 & $-0.268(0.168)$ & $-0.076(0.109)$ & $-0.056(0.140)$ \\
\hline Fame $2 *$ They & -0.023 & 0.041 & -0.020 & $-0.046(0.052)$ & $0.020(0.027)$ & $0.006(0.028)$ \\
\hline Fame $3 *$ They & 0.038 & $0.057 *$ & 0.063 & $-0.164(0.115)$ & $-0.093 * *(0.047)$ & $-0.0156 * *(0.050)$ \\
\hline Fame $1 *$ Ins & -0.067 & -0.055 & -0.057 & $-0.008(0.085)$ & $-0.060(0.052)$ & $-0.105^{\dagger}(0.059)$ \\
\hline Fame $2 *$ Ins & $0.073 * * *$ & 0.004 & $0.103 * *$ & $0.038(0.033)$ & $-0.006(0.013)$ & $-0.003(0.014)$ \\
\hline Fame $3 *$ Ins & $0.078 * * *$ & $0.050 * *$ & $0.082 *$ & $-0.132(0.097)$ & $-0.116 * *(0.032)$ & $-0.112 * *(0.042)$ \\
\hline Fame $1 *$ Cause & -0.099 & $-0.097 *$ & -0.105 & $-0.041(0.045)$ & $-0.072^{\dagger}(0.039)$ & $-0.045(0.038)$ \\
\hline Fame $2 *$ Cause & $-0.068 * * *$ & $-0.060 * * *$ & $-0.069 * *$ & $-0.065 * *(0.018)$ & $-0.052 * *(0.009)$ & $-0.052 * *(0.010)$ \\
\hline Fame $3 *$ Cause & -0.003 & $0.037 *$ & 0.006 & $0.079(0.158)$ & $-0.065 * *(0.029)$ & $0.062(0.061)$ \\
\hline Fame $1 *$ Disc & 0.499 & 0.050 & 0.010 & $-0.058(0.086)$ & $0.019(0.061)$ & $0.075(0.070)$ \\
\hline Fame $2 *$ Disc & $-0.060 * *$ & -0.023 & -0.048 & $-0.067 *(0.030)$ & $0.009(0.017)$ & $-0.028(0.017)$ \\
\hline Fame $3 *$ Disc & $0.073 * * *$ & 0.018 & $0.088 *$ & $1.709(2.705)$ & $0.056(0.054)$ & $-0.073(0.051)$ \\
\hline Fame $1 *$ Cert & 0.026 & 0.131 & 0.025 & $3.049(9.901)$ & $0.121(0.082)$ & $0.046(0.081)$ \\
\hline Fame 2: $*$ Cert & $0.253 * * *$ & $0.226 * * *$ & $0.270 * * *$ & $0.076(0.057)$ & $-0.018(0.018)$ & $0.009(0.022)$ \\
\hline Fame $3 *$ Cert & $0.149 * * *$ & $0.150 * * *$ & $0.165 * * *$ & $0.106(0.272)$ & $0.038(0.061)$ & $0.113(0.087)$ \\
\hline (Intercept) & $2.438 * * *$ & $-0.309 * * *$ & $0.441 * * *$ & $-0.311 * *(0.005)$ & $-1.198 * *(0.005)$ & $-2.219 * *(0.007)$ \\
\hline $\mathrm{N}$ & $1,541,354$ & 904,876 & 406,541 & \multicolumn{3}{|c|}{$\begin{array}{c}3,172,976 \\
\end{array}$} \\
\hline Log Likelihood & $-6,972,172$ & $-3,408,070$ & $-2,285,288$ & $-2,166,862$ & $-1,838,134$ & $-1,186,502$ \\
\hline AIC & $13,944,434$ & $6,816,231$ & $4,570,668$ & $4,333,812$ & $3,676,356$ & $2,373,092$ \\
\hline Theta & 0.115 & 0.153 & 0.031 & & & \\
\hline Std. Error & 0.0001 & 0.0002 & 0.0001 & & & \\
\hline \multicolumn{7}{|c|}{ 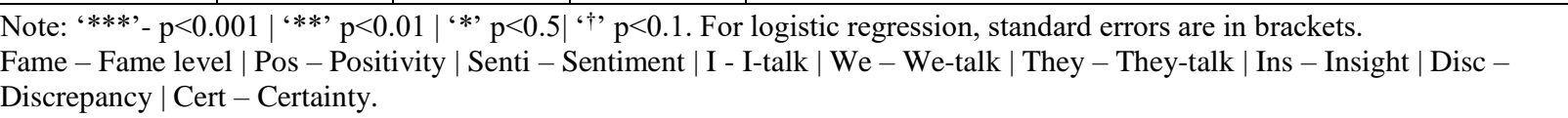 } \\
\hline
\end{tabular}




\section{Estimation results}

\subsection{Overall sample}

To assess the importance of affective and cognitive characteristics of tweets on engagement, we utilized negative binomial regressions, because our dependent variables, i.e., number of likes, comments, and retweets, are count data and are overdispersed (with $\chi^{2}(1)_{\text {Likes, }}, \chi^{2}(1)_{\text {Comments, }} \chi^{2}(1)_{\text {Retweets }}>1,000$ and p-values $<<0.0001$ respectively) [44]. The results for the overall sample are presented in Table 1. For message characteristics that engage via a peripheral route, we observe negative relationships between positivity and all three measures of engagement (model 1,2,3), confirming negativity bias as assumed in H1. Contrary to our expectations and the results of past studies (e.g., [10]), degree of emotional charge (i.e., sentiment) is negatively related to the number of likes, comments, and retweets, rejecting $\mathrm{H} 2$.
Next, the results suggest the significance of the variables measuring prosocial orientation. The socalled "we-talk" receives more likes and comments on the overall sample but does not significantly impact sharing behavior in terms of retweets (H3 partially supported). The use of $1^{\text {st }}$ person singular pronouns ("I-talk") is significantly negatively related to audience engagement.

We observe the significance of the cues processed via a central route. Interestingly, tweets expressing new insights received fewer likes, comments, and retweets (H4 rejected). Signs of certainty are also negatively related to the amount of audience involvement (H6 rejected). Providing causes and effects and spotting discrepancy is positively associated with likes, comments, and sharing content, supporting $\mathrm{H} 5$ and $\mathrm{H} 7$ on the overall sample.

To validate our findings, we also ran logistic regressions, with binary dependent variables that reflect whether a tweet has received at least one like (model 4), at least one comment (model 5), and at least one retweet (model 6). The results are consistent with the above-observed dynamics on counts.

Table 2. Regression estimates for the subsample of famous Twitter accounts

\begin{tabular}{|c|c|c|c|c|c|c|}
\hline Subsample & \multicolumn{3}{|c|}{ Famous } & \multicolumn{3}{|c|}{ Very famous } \\
\hline Model & $(7)$ & $(8)$ & $(9)$ & $(10)$ & $(11)$ & $(12)$ \\
\hline Method & \multicolumn{3}{|c|}{ Negative binomial regression } & \multicolumn{3}{|c|}{ Negative binomial regression } \\
\hline $\mathrm{DV} \rightarrow$ & LIKE & $\mathrm{COM}$ & RET & LIKE & $\mathrm{COM}$ & RET \\
\hline Positivity & $-0.090 * * *$ & $-0.170 * * *$ & $-0.087 * * *$ & $-0.055^{\dagger}$ & $-0.131 * * *$ & $-0.060 *$ \\
\hline Sentiment & $0.044 *$ & $0.018 * * *$ & $0.054 *$ & -0.014 & -0.027 & 0.005 \\
\hline I-talk & $0.226 * * *$ & $0.172 * * *$ & $0.196 * * *$ & $0.190 * * *$ & $0.138 * * *$ & $0.161 * * *$ \\
\hline We-talk & $0.130 * * *$ & $0.064 * * *$ & $0.100 * * *$ & $0.140 * * *$ & $0.064 * * *$ & $0.112 * * *$ \\
\hline They-talk & 0.005 & 0.024 & $0.028^{\dagger}$ & -0.028 & -0.010 & -0.007 \\
\hline Insight & $0.019 *$ & $0.025 * *$ & $0.014^{\dagger}$ & 0.013 & $0.020^{\dagger}$ & 0.013 \\
\hline Cause & $-0.031 * * *$ & $-0.020 * *$ & $-0.023 * * *$ & $-0.028 * *$ & -0.013 & $-0.022 *$ \\
\hline Discrepancy & $0.049 * * *$ & $0.070 * * *$ & $0.067 * * *$ & $0.024 *$ & $0.046^{* * *}$ & $0.032 * *$ \\
\hline Certainty & $0.146^{* * *}$ & $0.128 * * *$ & $0.162 * * *$ & $0.134 * * * *$ & $0.110 * * *$ & $0.139 * * *$ \\
\hline N hashtags & $-0.246 * * *$ & $-0.406 * * *$ & $-0.200 * * *$ & $-0.256 * * *$ & $-0.420 * * *$ & $-0.264 * * *$ \\
\hline URL & $-2.487 * * *$ & $-2.013 * * *$ & $-2.355 * * *$ & $-1.937 * * *$ & $-1.547 * * *$ & $-1.854 * * *$ \\
\hline WC & 0.002 & $0.003^{\dagger}$ & $0.006^{* *}$ & $-0.011 * * *$ & $-0.010 * * *$ & $-0.008 * *$ \\
\hline User activity & $0.005 * * *$ & $0.005 * * *$ & $0.005 * * *$ & $-0.004 * * *$ & $-0.002 * * *$ & $-0.004 *$ \\
\hline (Intercept) & $8.170 * * *$ & $5.631 * * *$ & $6.047 * * *$ & $9.322 * * *$ & $6.707 * * *$ & $7.269 * * *$ \\
\hline $\mathrm{N}$ & \multicolumn{3}{|c|}{9,050} & \multicolumn{3}{|c|}{3,354} \\
\hline Log Likelihood & $-58,146$ & $-36,543$ & $-41,906$ & $-26,321$ & $-34,700$ & $-19,983$ \\
\hline AIC & 116,323 & 73,117 & 83,842 & 52,673 & 36,117 & 39,997 \\
\hline Theta & 0.289 & 0.277 & 0.276 & 0.396 & 0.391 & 0.412 \\
\hline Std. Error & 0.003 & 0.004 & 0.004 & 0.008 & 0.008 & 0.008 \\
\hline
\end{tabular}




\subsection{Sub-sample of famous people}

In the next step, we extended our analysis by looking at the subsample of tweets made from accounts marked as famous. The rationale behind it is the presumably higher impact of celebrities, especially politicians, who may craft their content differently from the general public. In this vein, the tweets of participants with fame level values of 1,2 , and 3 were examined separately. Table 2 presents the regression estimates for the subsample of all people on the Socialbakers list, coined as "famous," and separately for people with more than 1,000,000 followers, coined as "very famous."

Consistent with the overall sample, the findings suggest that positivity is negatively related to engagement. Noteworthy, for the subsample of all tweets made by authors considered as famous, we observe the positive association between sentiment and engagement and no association for the subsample of "very famous" people (i.e., with more than 1,000,000 followers). As in the whole sample, "we-talk" is appreciated, generating more likes, comments, and retweets. At the same time, the use of single $1^{\text {st }}$ person pronouns also evidenced a positive relationship to engagement. As for the cognitive dimension, famous people can engage their audience on Twitter by spotting discrepancies and expressing certainty. The effect of expressed insights in tweets is insignificant for the information's diffusion as operationalized by the number of retweets. Pointing to a cause in posts is negatively related to the audience engagement for the subsample of famous participants. To sum up, the subsample of tweets from famous personalities exhibits differences in the effects of message characteristics on audience feedback compared to the overall sample. We discuss the findings together with their implications in the next section.

\section{Discussion and concluding remarks}

Politicians constantly try to convince citizens that their party has the "best solutions for the country's problems and the best people to implement those solutions" [45, p. 137]. This study sought to advance our understanding of how the public comes to react to these persuasive messages with its engagement on social media. Leaning on the ELM, we shed light on the effectiveness of affective (peripheral route) and cognitive (central route) cues on information appeal and dissemination on Twitter during a political event.

The results from the large-scale analysis of tweets during the 2020 U.S. presidential elections suggest the significance of both routes when processing a SM message. Specifically, we show that for the overall sample, the positivity of a tweet is negatively related to the number of likes, comments, and retweets it collects, which is in line with [3]. The core explanation behind these relationships is negativity bias leading masses to react stronger to the adverse impulses.

While emotionally charged content is reported to be accepted and spread more [6][7][10], in our sample, we observe the reverse pattern. Appreciation of less emotionally intensive posts may hint at an aversion towards extreme statements by the participants on election day. The reasons could vary from fatigue by the tension involved in the election to the attribution of strong claims to propaganda, which fuel polarization in society or might remain empty promises. Next, the prosocial orientation ("we-talk") expressed in a tweet drive SNS peers' likes and comments. It might be linked to the increased feeling of togetherness during a collective action like elections, the outcome of which defines the country's economic and general development vector for the next several years. In contrast, "I-talk" has the opposite effect.

On the central route, the significance of expressed discrepancy (i.e., a vision of how things should be) is remarkable. One possible rationale behind it is the appeal of a strategic plan as a property of a good leader whom the electorate can trust and rely on [46].

The post-hoc analysis on the subsample of people with large followings, i.e., thousands and sometimes millions of followers, delivered noteworthy insights. Here, we observe that positivity expressed in a tweet is still inversely related to engagement. At the same time, emotionally charged posts are liked, commented, and shared more, in contrast to the overall sample. However, for the top celebrities, the sentiment of a tweet does not significantly impact audience engagement. Thus, the focus should be shifted to other characteristics. Interestingly, both "I-talk" and "we-talk" generate likes, replies, and retweets, pointing to the importance of expressing belongingness as well as leadership and responsibility for their own opinion for famous people. On the cognitive route, this corresponds to the positive impact of certainty and discrepancy expressed in a Twitter post on peer support, feedback, and willingness to spread the word. Overall, these findings make theoretical and practical contributions and suggest avenues for future research.

For academics, we add to research on the impact of message characteristics in mediated online conversations [9][10][11] by empirically validating the "sentiment - public engagement" pathway in the political domain. While previously affective signals were in the spotlight, we refer to ELM [13][15] to conceptually account for the central and peripheral processing of a message and exhibit the significance of both paths. This study's results for a political event 
support the ELM relevance, earlier observed in other contexts (e.g., web personalization [13]). On a broader scale, our insights inform the dual-process theories in social psychology [47] and in the field of political persuasion [48], which as well rely on the cognitiveaffective dichotomy [49]. Our findings also highlight the importance of distinguishing between Twitter dynamics for average users and prominent people in politics, media, and society, thus advancing the research stream about SM influencers [36]. The effect of message characteristics for influential people on engagement differs, sometimes evidencing the opposite pattern than for average users.

As for practice, our findings suggest that to gain recognition in terms of likes, comments, and retweets, users might want to formulate their messages in a prosocial manner and express a vision or a plan, what is needed or should be improved. For celebrities, it might be worth being bold and, apart from "we-talk," also use "I-talk" to boost audience engagement.

Our study has several limitations, which may open interesting research directions. First, the data is limited to one political event and 24 hours (i.e., election day) time frame. Further studies may validate the results on a series of cases and on data for a longer time span, which in turn would allow accounting for the actual timing of tweets. Second, images, emojis, and videos in tweets were not a part of the current analysis. Third, our data set did not directly account for the exact number of followers a given poster had. While we mitigated this downside by assigning over 500 accounts from relevant celebrities, news entities, and politicians' levels according to their follower numbers, past research [10] has shown that the concrete number of followers significantly influences the number of interactions that posts achieve. This additional data might be informative in determining from which follower number onwards the observed pattern changes occur.

\section{References}

[1] Boulianne, S., "Social media use and participation: A meta-analysis of current research", Information, Communication \& Society, 18(5), 2015, pp. 524-538.

[2] Valenzuela, S., "Unpacking the use of social media for protest behavior: The roles of information, opinion expression, and activism", American Behavioral Scientist, 57(7), 2013, pp. 920-942.

[3] Mallipeddi, R. R., Janakiraman, R., Kumar, S., and Gupta, S. "The Effects of Social Media Content Created by Human Brands on Engagement: Evidence from Indian General Election 2014”, Information Systems Research, 32(1), 2021, pp. 212-237.

[4] Stieglitz, S., Mirbabaie, M., Schwenner, L., Marx, J., Lehr, J., and Brünker, F., "Sensemaking and Communication Roles in Social Media Crisis Communication", In
Proceedings of the 13th International Conference for Wirtschaftsinformatik, St. Gallen, 2017, pp. 1333-1347.

[5] Tumasjan, A., Sprenger, T., Sandner, P., and Welpe, I. "Predicting elections with twitter: What 140 characters reveal about political sentiment", In Proceedings of the International AAAI Conference on Web and Social Media (Vol. 4, No. 1), 2010, pp. 178-185.

[6] Berger, J., and Milkman, K. L. "What makes online content viral?", Journal of Marketing Research, 49(2), 2012, pp. 192-205.

[7] Heimbach, I., and Hinz, O. "The impact of content sentiment and emotionality on content virality", International Journal of Research in Marketing, 33(3), 2016, pp. 695-701.

[8] Faralli, S., Rittinghaus, S., Samsami, N., Distante, D., and Rocha, E., "Emotional intensity-based success prediction model for crowdfunded campaigns", Information Processing \& Management, 58(1), 2021, 102394.

[9] Chen, L., Baird, A., and Straub, D. "A linguistic signaling model of social support exchange in online health communities", Decision Support Systems, 130, 2020, 113233.

[10] Stieglitz, S., and Dang-Xuan, L. "Emotions and information diffusion in social media-sentiment of microblogs and sharing behavior", Journal of Management Information Systems, 29(4), 2013, pp. 217248.

[11] Gorodnichenko, Y., Pham, T., and Talavera, O. "Social media, sentiment and public opinions: Evidence from \# Brexit and \# USElection", European Economic Review, 2021, 103772.

[12] Trottier, D., and Fuchs, C. (Eds.). "Social media, politics and the state: protests, revolutions, riots, crime and policing in the age of Facebook, Twitter and YouTube", Routledge, 2014.

[13] Tam, K. Y., and Ho, S. Y., "Web personalization as a persuasion strategy: An elaboration likelihood model perspective", Information Systems Research, 16(3), 2005, pp. 271-291.

[14] Ashman, M., Introduction to Professional Communications, 2018.

[15] Humphreys, A. R., "Causation, complexity, and the concert: the pragmatics of causal explanation in international relations", Journal of International Relations and Development, 20(4), 2017, pp. 717-736.

[16] Petty, R. E., and Cacioppo, J. T., "The elaboration likelihood model of persuasion", Advances in Experimental Social Psychology, 19, 1986, pp. 123-205.

[17] Xu, W. W., and Zhang, C., "Sentiment, richness, authority, and relevance model of information sharing during social Crises - the case of \#MH370 tweets", Computers in Human Behavior, 89, 2018, pp. 199-206.

[18] Hanson R, https://www.rickhanson.net/, 2021.

[19] Soroka, S., Fournier, P., and Nir, L., "Cross-national evidence of a negativity bias in psychophysiological reactions to news", In Proceedings of the National Academy of Sciences, 116(38), 2019, pp. 18888-18892.

[20] Abramova, O., Shavanova, T., Fuhrer, A., Krasnova, H., and Buxmann, P., "Understanding the sharing economy: The role of response to negative reviews in the peer-to- 
peer accommodation sharing network", In Proceedings of the European Conference in Information Systems, 2015.

[21] Stieglitz, S., and Dang-Xuan, L., "Impact and diffusion of sentiment in public communication on Facebook", 2012.

[22] Kelley, H. H., and Thibaut, J. W., Interpersonal relations: A theory of Interdependence, Wiley, New York, 1978.

[23] Karan, A., Rosenthal, R., and Robbins, M. L., "Metaanalytic evidence that we-talk predicts relationship and personal functioning in romantic couples", Journal of Social and Personal Relationships, 36(9), 2019, pp. 26242651.

[24] Kacewicz, E., Pennebaker, J. W., Davis, M., Jeon, M., and Graesser, A. C., "Pronoun use reflects standings in social hierarchies", Journal of Language and Social Psychology, 33(2), 2014, pp. 125-143.

[25] Lewiński, M., and Mohammed, D., "Argumentation in political deliberation", Journal of Argumentation in Context, 2(1), 2013.

[26] Wagner, A., Abramova, O., Krasnova, H., and Buxmann, P, "When You Share, You Should Care: Examining the Role of Perspective-Taking on Social Networking Sites", In Proceedings of the European Conference of Information Systems National Academy of Sciences, 2018.

[27] Intyaswati, D., Maryani, E., Sugiana, D., and Venus, A, "Social Media as an Information Source of Political Learning in Online Education", SAGE Open, 11(2), 2021, 21582440211023181.

[28] Lane, D. S., Kim, D. H., Lee, S. S., Weeks, B. E., and Kwak, N, "From online disagreement to offline action: How diverse motivations for using social media can increase political information sharing and catalyze offline political participation", Social Media + Society, 3(3), 2013, 2056305117716274.

[29] Jost, J. T., Barberá, P., Bonneau, R., Langer, M., Metzger, M., Nagler, J., ... and Tucker, J. A., "How social media facilitates political protest: Information, motivation, and social networks", Political Psychology, 2018, 39, pp. 85118.

[30] Brady, H. E., Goodin R. E. (Ed.), Causation and explanation in social science, 2008, pp. 217-70.

[31] Tobin, S. J., and Raymundo, M. M., "Persuasion by causal arguments: The motivating role of perceived causal expertise", Social Cognition, 2009, 27(1), pp. 105127.

[32] Hunt, J. M., Domzal, T. J., and Kernan, J. B, "Causal attributions and persuasion: The case of disconfirmed expectancies", ACR North American Advances, 1982.

[33] Rosenthal, S. A., Pittinsky, T. L., Purvin, D. M., and Montoya, R. M., "National Leadership Index 2007: A national study of confidence in leadership", US News and World Report, 2007.

[34] Bail, C. A., Argyle, L. P., Brown, T. W., Bumpus, J. P., Chen, H., Hunzaker, M. F. and Volfovsky, A., "Exposure to opposing views on social media can increase political polarization", In Proceedings of the National Academy of Sciences, 115(37), 2018, 9216-9221.

[35] Zaccaro, S. J., and Banks, D. J. "Leadership, vision, and organizational effectiveness", In: The Nature of Organizational Leadership: Understanding the
Performance Imperatives Confronting Today's Leaders, 2001, pp. 181-218.

[36] Chae, J., "Explaining females' envy toward social media influencers", Media Psychology, 21(2), 2018, pp. 246262.

[37] Shuyo, N., Language Detection Library for Java, 2010, https://github.com/shuyo/language-detection.

[38] Thelwall, M., Buckley, K., Paltoglou, G. Cai, D., and Kappas, A., "Sentiment strength detection in short informal text", Journal of the American Society for Information Science and Technology, 61(12), 2010, pp. $2544-2558$.

[39] Thelwall, M., Buckley, K., and Paltoglou, G., "Sentiment strength detection for the social Web", Journal of the American Society for Information Science and Technology, 63(1), 2012, pp. 163-173.

[40] Koto, F., and Adriani, M, "A comparative study on twitter sentiment analysis: Which features are FFgood?", In: International Conference on Applications of Natural Language to Information Systems, 2015, Springer, Cham, pp. 453-457.

[41] Pennebaker, J. W., Boyd, R. L., Jordan, K., and Blackburn, K, "The development and psychometric properties of LIWC2015", Austin, TX: University of Texas at Austin, 2015.

[42] Socialbakers, https://www.socialbakers.com/, 2021.

[43] Suh, B., Hong, L., Pirolli, P., and Chi, E. H. "Want to be retweeted? large scale analytics on factors impacting retweet in twitter network", In 2010 IEEE Second International Conference on Social Computing, 2010, pp. 177-184.

[44] Cameron, A.C., and Trivedi, P.K. Regression Analysis of Count Data. Cambridge: Cambridge university Press, 1998.

[45] Kerkhof, P., "Applying the unimodel to political persuasion", Psychological inquiry, 10(2), 1999, pp. 137-140.

[46] Antonakis, J., Fenley, M., and Liechti, S. "Can charisma be taught? Tests of two interventions", Academy of Management Learning \& Education, 10(3), 2011, pp. 374-396.

[47] Yu, J., Hu, P. J. H., and Cheng, T. H. "Role of affect in self-disclosure on social network websites: A test of two competing models", Journal of Management Information Systems, 32(2), 2015, pp. 239-277.

[48] Cobb, M. D., and Kuklinski, J. H., "Changing minds: Political arguments and political persuasion", American Journal of Political Science, 1997, pp. 88-121.

[49] See Y. H. M., "Cognitive-Affective Processing System." In: Zeigler-Hill V., Shackelford T. (Eds) Encyclopedia of Personality and Individual Differences. Springer, Cham, 2018. 